\title{
SMA numerical modeling versus experimental results
}

\author{
Ferdinando Auricchio ${ }^{123}$, Simone Morganti ${ }^{1}$, and Alessandro Reali ${ }^{123, \text { a }}$ \\ ${ }^{1}$ Dipartimento di Meccanica Strutturale (DMS), Università degli Studi di Pavia, Via Ferrata 1, 27100 \\ Pavia, Italy \\ 2 Istituto di Matematica Applicata e Tecnologie Informatiche (IMATI-CNR), Pavia, Italy \\ ${ }^{3}$ European Centre for Training and Research in Earthquake Engineering (EUCENTRE), Pavia, Italy
}

\begin{abstract}
The unique features characterizing shape memory alloys (SMAs), such as superelasticity and shape memory effect, stimulate a deep investigation that aims at fully understanding their behavior through experimental tests. In the present work, we compare numerical results obtained using the phenomenological model proposed by Auricchio and Petrini in 2004 with some experiments carried out on SMA wires within S3T Roundrobin SMA modeling activities.
\end{abstract}

\section{Introduction}

In this work, we compare numerical results obtained using the phenomenological model proposed by Auricchio and Petrini in [2] with some experiments carried out on SMA wires within S3T Roundrobin SMA modeling activities.

We introduce the employed model and its basic equations. We then show the parameter identification procedure we use and present the parameters that we adopt in all performed numerical simulations. Finally, we simulate different experiments, showing comparisons between numerical and experimental results. We also propose some concluding remarks highlighting the main observed points of strength and weakness of the considered model.

\section{Model basic equations}

The set of constitutive equations capable of describing SMA macroscopic main behaviors are deduced on the basis of the constitutive model presented in [2], taking its inspiration by the work of Souza et al. [6]. Assuming a small strain regime, the expression of the free-energy density function $\Psi$ for a polycrystalline SMA material can be defined through the following convex potential

$$
\Psi\left(\theta, \boldsymbol{e}, \boldsymbol{e}^{t r}, T\right)=\frac{1}{2} K \theta^{2}+G\left\|\boldsymbol{e}-\boldsymbol{e}^{t r}\right\|^{2}+\beta\left(T-T^{*}\right)^{+}\left\|\boldsymbol{e}^{t r}\right\|+\frac{1}{2} h\left\|\boldsymbol{e}^{t r}\right\|^{2}+\mathcal{I}_{\varepsilon_{L}}\left(\boldsymbol{e}^{t r}\right),
$$

where: $\theta=\operatorname{tr}(\varepsilon)$ and $\boldsymbol{e}$ are respectively the volumetric and the deviatoric part of the total strain $\varepsilon$, while $\boldsymbol{e}^{t r}$ is the transformation strain; $K$ and $G$ are the bulk and the shear moduli; $\|\cdot\|$ is the Euclidean norm and $(\cdot)^{+}$the positive part function; $\beta$ is a material parameter related to the dependence of the critical stress on the temperature, $T^{*}$ is the temperature below which only martensite phase is stable, and $h$ is a material parameter related to the hardening of the

a e-mail: alessandro.reali@unipv.it 
material during the phase transformation. Finally, $\mathcal{I}\left(e^{t r}\right)$ is an indicator function introduced to satisfy the constraint on the transformation strain norm

$$
\mathcal{I}_{\varepsilon_{L}}\left(\boldsymbol{e}^{t r}\right)=\left\{\begin{aligned}
0 & \text { if }\left\|\boldsymbol{e}^{t r}\right\| \leq \varepsilon_{L} \\
+\infty & \text { otherwise }
\end{aligned}\right.
$$

where $\varepsilon_{L}$ is a material parameter related to the maximum transformation strain reached at the end of the transformation during an uniaxial test.

We remark that in the expression of the free energy we neglect the contributions due to thermal expansion and change in temperature with respect to the reference state, since we are not interested here in a complete description of the thermomechanical coupled problem. However, the interested reader may refer to $[2,3]$ to see how it is possible to take into account these aspects in the formulation. We also stress that, due to the fact that we do not consider a fully thermomechanical coupled model, $\Psi$ should be more properly referred to as a temperatureparameterized free-energy density function.

Following standard arguments, it is possible to compute the quantities thermodynamically conjugate to the volumetric and deviatoric components of the strain and to the transformation strain. In particular, we have

$$
\left\{\begin{aligned}
p & =\frac{\partial \Psi}{\partial \theta}=K \theta \\
s & =\frac{\partial \Psi}{\partial \boldsymbol{e}}=2 G\left(\boldsymbol{e}-\boldsymbol{e}^{t r}\right), \\
\boldsymbol{X} & =-\frac{\partial \Psi}{\partial \boldsymbol{e}^{t r}}=s-\beta\left(T-T^{*}\right)^{+} \frac{\boldsymbol{e}^{t r}}{\left\|\boldsymbol{e}^{t r}\right\|}-h \boldsymbol{e}^{t r}-\gamma \frac{\boldsymbol{e}^{t r}}{\left\|\boldsymbol{e}^{t r}\right\|}
\end{aligned}\right.
$$

where $p=\operatorname{tr}(\boldsymbol{\sigma}) / 3$ and $s$ are respectively the volumetric and the deviatoric part of the stress $\boldsymbol{\sigma}, \boldsymbol{X}$ is a thermodynamic stress-like quantity associated to the transformation strain $\boldsymbol{e}^{t r}$. The variable $\gamma$ results from the indicator function subdifferential $\partial \mathcal{I}_{\varepsilon_{L}}\left(\boldsymbol{e}^{t r}\right)$ and it is defined as

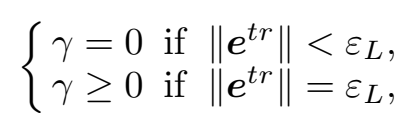

so that $\partial \mathcal{I}_{\varepsilon_{L}}\left(\boldsymbol{e}^{t r}\right)=\gamma \frac{\boldsymbol{e}^{t r}}{\left\|\boldsymbol{e}^{t r}\right\|}$.

To describe phase transformation and inelasticity evolution, we choose (following a plasticitylike terminology) a limit function $F$ defined as

$$
F(\boldsymbol{X})=\|\boldsymbol{X}\|-R,
$$

where $R$ is the radius of the elastic domain.

Considering an associative framework, the flow rules for the internal variables take the form

$$
\dot{\boldsymbol{e}}^{t r}=\dot{\zeta} \frac{\partial F}{\partial \boldsymbol{X}}=\dot{\zeta} \frac{\boldsymbol{X}}{\|\boldsymbol{X}\|} .
$$

The model is finally completed by the classical Kuhn-Tucker conditions

$$
\left\{\begin{array}{c}
\dot{\zeta} \geq 0, \\
F \leq 0, \\
\dot{\zeta} F=0 .
\end{array}\right.
$$

\section{Parameter identification}

The identification of the material parameters is easily achieved through a comparison between two thermal cycling tests at constant stress, in a proportional 1D setting, as described in [1]. 
In fact, as highlighted in Figure 1 (left), $\varepsilon_{L}$ is immediately obtained. $E$ and $\beta$ can be computed, using their definition, respectively as

$$
E=\frac{\Delta \sigma}{\Delta \varepsilon} \text { and } \beta=\frac{\Delta \sigma}{\Delta T}
$$

with $\Delta \varepsilon, \Delta T=T_{y M}^{(1)}-T_{y M}^{(2)}$ and $\Delta \sigma=\sigma^{(1)}-\sigma^{(2)}$ as indicated in Figure 1 . Then, it is possible to compute $R$ knowing that the hysteresis width is $2 R / \beta$ (see again Figure 1 ). The equation $\left(T_{y A}^{(1)}-T_{y M}^{(1)}\right)=2 R / \beta$ is obtained subtracting the two following equations (deriving from $F=$ $|X|-R=0)$

$$
\left\{\begin{array}{l}
\sigma-\beta\left(T_{y M}-T^{*}\right)=R \\
\sigma-\beta\left(T_{y A}-T^{*}\right)=-R
\end{array}\right.
$$

Moreover, starting from $\frac{\partial \sigma}{\partial \varepsilon}=\frac{E h}{E+h}$ and considering the dependence of the stress on temperature $\left(\frac{\partial \sigma}{\partial \varepsilon}=\frac{\partial \sigma}{\partial T} \frac{\partial T}{\partial \varepsilon}=\beta \frac{\partial T}{\partial \varepsilon}\right)$, with simple computations it can be found that

$$
h=\frac{1}{\frac{1}{\beta} \frac{\partial \varepsilon}{\partial T}-\frac{1}{E}} .
$$

Finally, to compute $T^{*}$, the following expression, directly derived from $\sigma^{(1)}-\beta\left(T_{y M}^{(1)}-T^{*}\right)=$ $R$, can be used

$$
T^{*}=T_{y M}^{(1)}+\frac{R-\sigma^{(1)}}{\beta} .
$$

The employed $\varepsilon-T$ curves are those referred to thermal cycling tests at $400 \mathrm{MPa}$ and 450 $\mathrm{MPa}$ (see Figure 1, middle). In Figure 1 (right) we also show the obtained material parameters (already reported to a 3D setting), which we will use for the numerical simulations of all tests reported in the next section.

\section{Numerical results}

In this section we compare the numerical results obtained using the parameters identified in the previous sections with the experimental results provided on the Esomat webpage by the organizers of the S3T Roundrobin SMA modeling session ([7]).

\subsection{Tension tests}

In this section, we present simulations of tension tests. Figure 2 shows a comparison between experimental and numerical results in terms of $\sigma-\varepsilon$ at three different constant temperatures $T=-20,10,60^{\circ} \mathrm{C}$.

\subsection{Thermal cycling tests at constant stress}

In this section, we present simulations of thermal cycling tests at constant stress. Accordingly, Figure 3 reports $\varepsilon-T$ diagrams obtained cycling temperature at constant stress. Since we used two of these curves for the parameter identification procedure, the numerical $\varepsilon-T$ plot in Figure 3 (top) is partially fitted and partially predicted, while the $\varepsilon-T$ plot in Figure 3 (middle-top) is totally fitted; instead, $\varepsilon-T$ plots in Figure 3 (middle-bottom and bottom) are totally predicted. 


\subsection{Combined tension-torsion tests at constant stress}

In this section, a comparison between numerical and experimental tension-torsion results in terms of torque and axial strain as a function of the angular displacement is presented and reported in Figure 4. All tests are performed at a constant tensile stress of $70 \mathrm{MPa}$, but at different temperatures.

We highlight that the numerical simulation of this kind of tests requires the solution of a boundary value problem. In order to do that, we implemented the constitutive model within the finite element commercial code Abaqus, which we used to solve all studied tension-torsion boundary value problems.

\subsection{Combined tension-torsion tests at constant temperature}

Also in this section, a comparison between numerical and experimental tension-torsion results in terms of torque and axial strain as a function of the angular displacement is presented and reported in Figure 5, but here tests are performed for different tensile stresses at a constant temperature of $30^{\circ} \mathrm{C}$.

\subsection{Thermomechanical recovering stress tests}

We finally present simulations of thermomechanical recovering stress tests. The results presented in Figure 6 are carried out on a wire strained in tension at room temperature $\left(T=24^{\circ} \mathrm{C}\right)$ up to $3.5 \%$ prestrain, both at upper and lower plateau, followed by thermal cycling at constant prestrain and final unloading at room temperature.

\section{Concluding remarks}

The performed simulations reveal that the employed model, despite the very low number of used material parameters (i.e., only 7), is able to reproduce well, from a qualitative point of view, all the studied experimental data-sets with a unique choice of material parameters. We also highlight that the adopted constitutive model allows a simple and robust implementation within finite element codes, an important issue when dealing with the solution of complex boundary value problems (as it usually happens when studying real structural problems).

We moreover remark that the simulation results could be improved extending the model to include other effects, such as plasticity, transformation- and temperature-dependent material parameters, asymmetric behaviors, as indicated in $[4,5]$.

It is finally to be noted that quantitative agreement with experimental data is not good in some cases (e.g., in some torsion tests). We believe that this can be mainly due to the adopted J2-type yield surface with a constant radius. We finally remark that R-phase cannot be reproduced by the used model.

\section{Acknowledgements}

This work has been partially supported by the European Science Foundation through the EUROCORES S3T project "SMARTeR: Shape Memory Alloys to Regulate Transient Responses in civil engineering" and by the European Research Council through the Starting Independent Research Grant "BioSMA: Mathematics for Shape Memory Technologies in Biomechanics". 


\section{References}

1. F. Auricchio, A. Coda, A. Reali, M. Urbano, Journal of Materials Engineering and Performance, vol. 18, (2009) pp. 649-654

2. F. Auricchio, L. Petrini, International Journal for Numerical Methods in Engineering, vol. 61, (2004) pp. 807-836

3. F. Auricchio, L. Petrini, International Journal for Numerical Methods in Engineering, vol. 61, (2004) pp. 716-737

4. F. Auricchio, A. Reali, U. Stefanelli, International Journal of Plasticity, vol. 23, (2007) pp. 207-226

5. F. Auricchio, A. Reali, U. Stefanelli, Computer Methods in Applied Mechanics and Engineering, vol. 198, (2009) pp. 1631-1637

6. A. C. Souza, E. N. Mamiya, N. Zouain, European Journal of Mechanics, A: Solids, vol. 17, (1998) pp. $789-806$

7. Esomat S3T Roundrobin SMA modeling session,

http://esomat.fzu.cz/esomat2009/index.php/esomat/esomat2009/modelling_session.
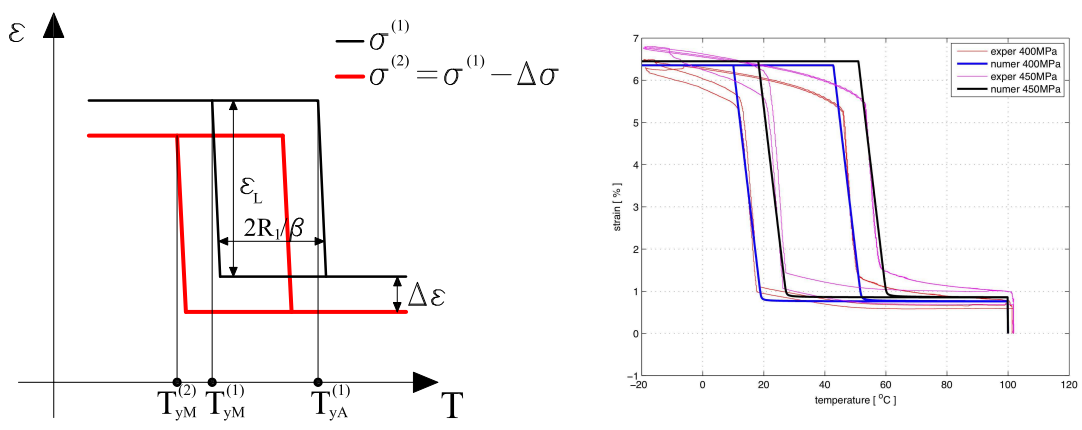

\begin{tabular}{|ll|}
\hline$E:$ & $53000 \mathrm{MPa}$ \\
$v:$ & 0.33 \\
$h:$ & $1000 \mathrm{MPa}$ \\
$\mathrm{T}^{*}:$ & $-30^{\circ} \mathrm{C}$ \\
$\beta:$ & $6.1 \mathrm{MPa} /{ }^{\circ} \mathrm{C}$ \\
$\mathrm{R}:$ & $100 \mathrm{MPa}$ \\
$\varepsilon_{\mathrm{L}}:$ & $5.6 \%$ \\
\hline
\end{tabular}

Fig. 1. Parameter identification procedure. Two strain-temperature curves at two constant stresses $\sigma^{(1)}>\sigma^{(2)}$ (left); strain-temperature experimental and numerical curves at 400MPa and $450 \mathrm{MPa}$ (middle); identified material parameters (right).
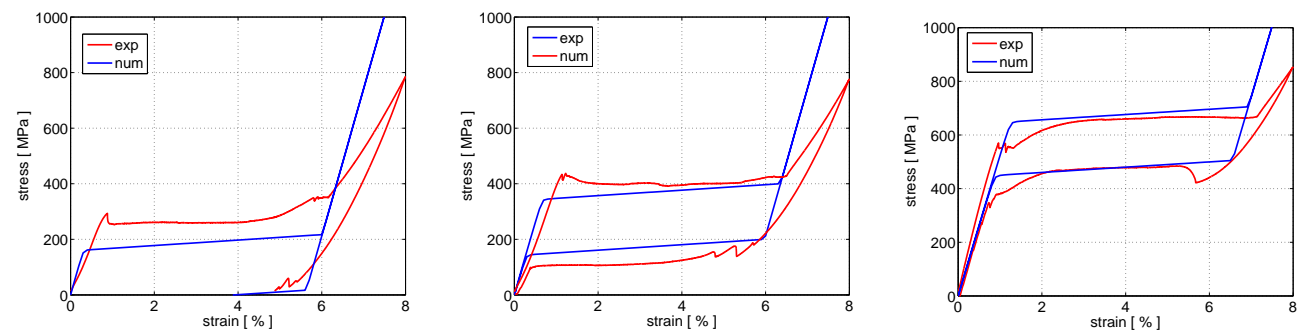

Fig. 2. $\sigma-\varepsilon$ curves at constant temperatures of, respectively (from left to right), $T=-20,10,60^{\circ} \mathrm{C}$.
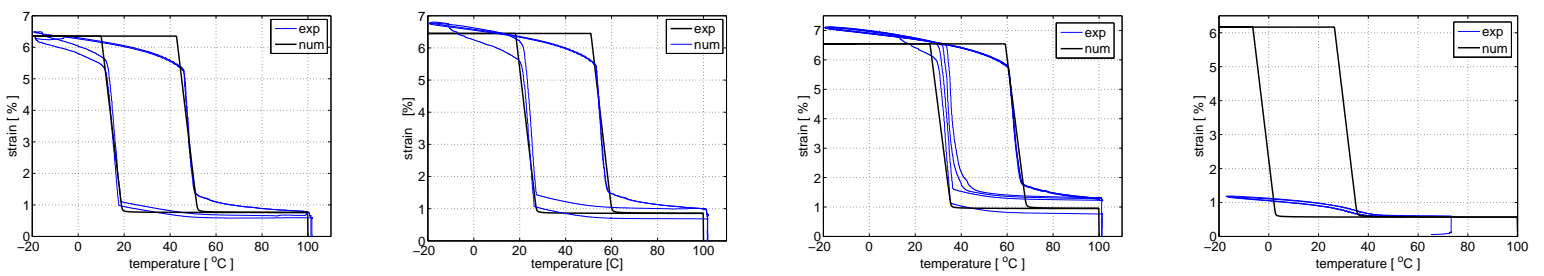

Fig. 3. $\varepsilon-T$ curves at constant tensile stresses of, respectively (from left to right), $\sigma=$ 400, 450, 500, 300MPa. 

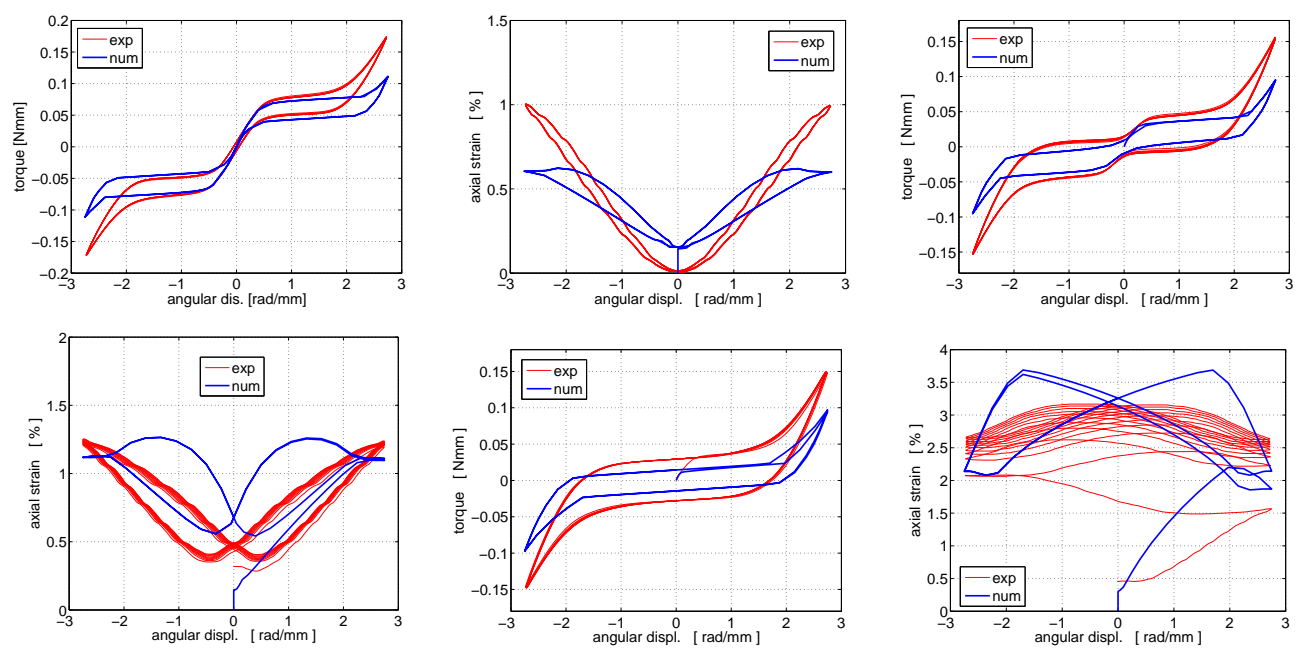

Fig. 4. Torque vs angular displacement and axial strain vs angular displacement curves in a combined tension-torsion test at $30^{\circ} \mathrm{C}$ (top, left and middle), at $-10^{\circ} \mathrm{C}$ (top right and bottom left), and at $-30^{\circ} \mathrm{C}$ (bottom, middle and right); the constant axial tension is equal to $70 \mathrm{MPa}$.
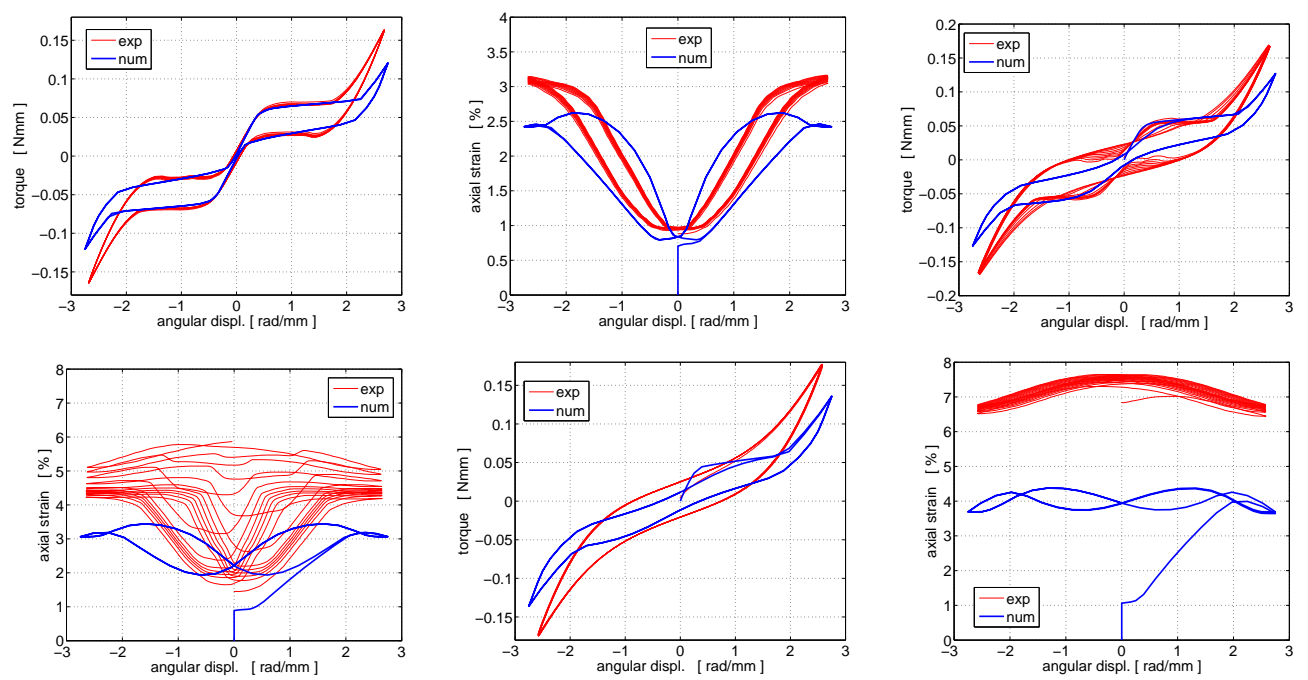

Fig. 5. Torque vs angular displacement (top) and axial strain vs angular displacement (bottom) curves in a combined tension-torsion test at $30^{\circ} \mathrm{C}$; the constant axial tension is respectively equal to 255 (top, left and middle), 317 (top right and bottom left), and 379 (bottom, middle and right) MPa.
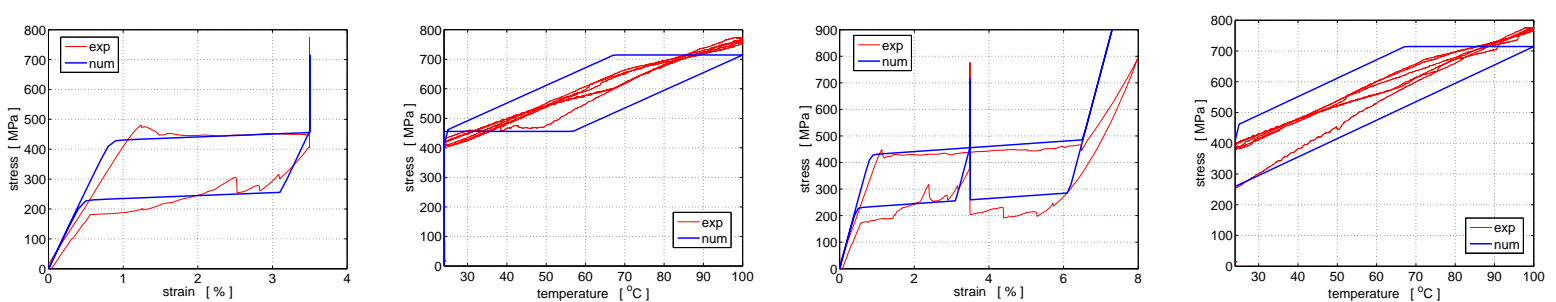

Fig. 6. $\sigma-\varepsilon$ and $T-\sigma$ curves obtained reaching $3.5 \%$ prestrain at the upper (two plots on the left) and at the lower (two plots on the right) plateau at room temperature $\left(24^{\circ} \mathrm{C}\right)$, then, cycling with temperature and, finally, unloading at room temperature. 